\title{
Evolvability of the mode of peptide binding by an RNA
}

TETSUYA IWAZAKI, XIANGLAN LI, and KAZUO HARADA

Department of Life Science, Tokyo Gakugei University, Koganei, Tokyo 184-8501, Japan

\begin{abstract}
The HIV Rev-response element (RRE) RNA binds strongly to two unrelated peptides, the HIV Rev peptide and an RRE-binding aptamer, the RSG-1.2 peptide, at a similar site, but using distinct sets of interactions. In this study, the nucleotide base requirements for the binding of the RRE to the Rev and RSG-1.2 peptides were determined by selection of Rev- and RSG-1.2binding RRE variants using a bacterial reporter system. As a result, distinct differences in the bases necessary for binding the two peptides were found in the upper stem of the RRE. Strikingly, single nucleotide changes in this region were found to switch the peptide-binding specificity of the RRE from a bifunctional Rev- and RSG-1.2-binding mode to either a Rev-specific or a RSG-1.2specific mode, demonstrating how an RNA can evolve alternative binding strategies in discrete steps without intermediate loss of function. This evolvability of the mode of peptide binding by an RNA presumably reflects the multidimensionality of conformational space that a given RNA has available for ligand recognition, which may have been utilized in the evolution of RNApolypeptide complexes.
\end{abstract}

Keywords: RNA-peptide interactions; RNA structure; in vivo RNA selection; bacterial reporter system; molecular evolution

\section{INTRODUCTION}

The arginine-rich RNA-binding motif is a class of short peptides 10-20 residues in length that bind to their RNA sites with affinities and specificities approaching those of the intact protein and has been a particularly useful model system for studying principles of RNA-protein interactions (Patel 1999; Frankel 2000; Cheng et al. 2001; Das and Frankel 2003). Although referred to as a motif, this class of peptides has no conserved structure and has been shown to bind in a variety of conformations, such as an $\alpha$-helix in the case of HIV Rev and phage N peptides (Tan et al. 1993; Battiste et al. 1996; Cai et al. 1998; Legault et al. 1998), a $\beta$ hairpin structure in the case of the bovine immunodeficiency virus (BIV) Tat peptide (Chen and Frankel 1995; Puglisi et al. 1995), and an extended conformation in the case of the HIV Tat peptide (Calnan et al. 1991; Aboul-ela et al. 1995). The RNA sites to which these peptides bind are also diverse and are generally located at bulged and looped regions within relatively structured RNAs (Heaphy et al. 1991; Tiley et al. 1992; Chen and Frankel 1994), where the narrow major groove of the RNA double helix is widened,

Reprint requests to: Kazuo Harada, Department of Life Science, Tokyo Gakugei University, Koganei, Tokyo 184-8501, Japan; e-mail: harada@ u-gakugei.ac.jp; fax: 81-42-329-7550.

Article published online ahead of print. Article and publication date are at http://www.rnajournal.org/cgi/doi/10.1261/rna.2560905. therefore allowing recognition of the bases (Weeks and Crothers 1993). Adaptive binding involving unstructured to structured transitions of peptide and/or RNA upon complex formation has also been found to be a general feature (Frankel and Smith 1998; Patel 1999).

A short peptide corresponding to the RNA-binding domain of the HIV Rev protein, the Rev peptide, has been shown to bind to an RNA structure within the HIV genome referred to as the Rev-response element (RRE) (Fig. 1). Mutagenesis and chemical modification experiment showed that the Rev peptide binds to an internal loop region of the RRE consisting of G46-C51 and U66-C74 of the RRE (Fig. 1A; Kjems et al. 1991, 1992; Tiley et al. 1992). In vitro selection of RRE variants that bind to Rev resulted in the elucidation of a consensus structure that matched the above results and also led to the identification of RRE variants with a compensatory G48A/G71A substitution, suggesting the presence of a noncanonical G48-G71 base pair that can be replaced by an isostructural A48-A71 base pair (Fig. 1A; Bartel et al. 1991). The NMR structure of the Rev-RRE complex later showed that the formation of the G48-G71, as well as a G47-A73, base pair results in the widening of an otherwise narrow major groove, allowing the binding of the $\alpha$-helical Rev peptide deep into the RNA major groove (Battiste et al. 1996).

On the other hand, a number of novel RRE-binding peptides have been identified from random peptide 


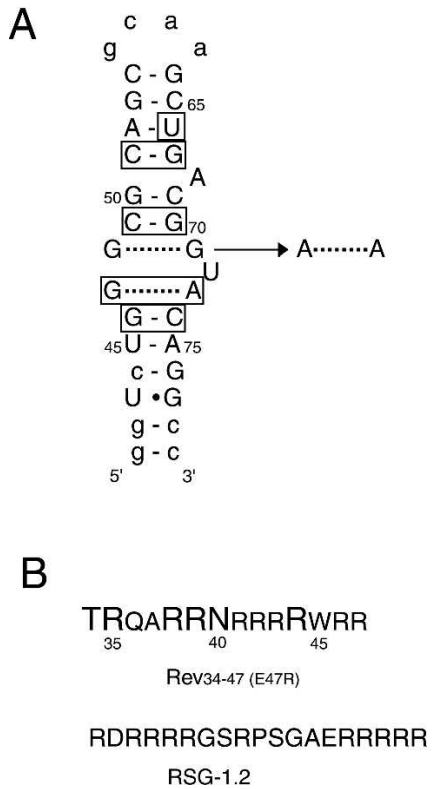

FIGURE 1. The secondary structure of the RRE and the sequence of RRE-binding peptides. (A) The secondary structure of the RRE IIB. Boxed nucleotides are those known to be important for Rev binding. Large letters indicate wild-type RRE nucleotides, while small letters indicate those that have been modified. $(B)$ The amino acid sequence of the RNA-binding domain of the Rev peptide and that of a selected RRE-binding aptamer, RSG-1.2. The large letters in the Rev peptide indicate those amino acids found to be important for RRE binding.

libraries, showing that multiple strategies exist for the recognition of the same RNA site (Harada et al. 1996, 1997; Friesen and Darby 1998; Tan and Frankel 1998; Peled-Zehavi et al. 2003). In one such selection experiment using a bacterial reporter assay for detecting RNA-polypeptide interactions, RRE-binding peptides were selected from a randomized library consisting of three amino acids $(\mathrm{R}, \mathrm{S}$, and $G$ ), then further evolved by mutagenesis and reselection, resulting in the identification RSG-1.2 peptide, which bound to the RRE several times more strongly and specifically than the Rev peptide (Fig. 1B; Harada et al. 1996, 1997). NMR structural analysis of the RRE-RSG-1.2 complex showed that the peptide binds deeply into the characteristic widened major groove of the RRE, as observed in the Rev-RRE complex (Gosser et al. 2001; Zhang et al. 2001). However, while the helical Rev peptide binds along the major groove of the RRE in a fashion similar to the binding of $\alpha$-helices to the major groove of doublestranded DNA, the RSG-1.2 peptide binds in an unstructured-turn-helix peptide conformation and with the helix axis of the peptide almost perpendicular to that of the RRE. Examination of the details of the interaction showed that a considerably different set of interactions appeared to be used for RNA-peptide recognition. In the case of Rev, four specific amino acid side chain-nucleotide base interactions, an asparagine amide group (N40) binding to the G47-A73 and the arginine guanidinium groups of R35,
R39, and R44 binding to G67, G70, and U45, respectively, along with several arginine guanidinium-phosphate interactions and a number of hydrophobic interactions, were observed (Battiste et al. 1996). On the other hand, in the case of RSG-1.2, only two side chain-base interactions involving arginine guanidinium groups binding to G70 and A73 were observed, while extensive hydrophobic interactions involving alanine and proline residues appeared to be important in recognizing the shape of the RNA major groove (Gosser et al. 2001; Zhang et al. 2001).

In this study, the nucleotide base requirements for the binding of the RRE to Rev and RSG-1.2 were determined by selection of Rev- and RSG-1.2-binding RRE variants using a bacterial reporter system. Interestingly, we found that the selected RRE variants could be classified into a number of groups depending on their specificity toward the RSG-1.2 and Rev peptides and that distinct differences in the bases necessary for binding the two peptides existed in the upper stem of the RRE. Further analysis showed that the specificity of the RRE toward the RSG-1.2 and Rev peptides could be altered by single nucleotide substitutions, demonstrating the ease with which the mode of peptide binding by an RNA can be evolved.

\section{RESULTS}

\section{In vivo selection of RSG-1.2-binding RRE variants}

RSG-1.2-binding RRE variants were selected from a doped RRE library using a bacterial two-plasmid system for detecting RNA-polypeptide interactions (Fig. 2; Harada et al. 1996; Peled-Zehavi et al. 2003). A DNA cassette encoding a library of RRE variants, in which a 26-nucleotide region that included U45-C54 and G64-A75 of the wild-type sequence and a GCAA tetraloop cap (Fig. 1A)

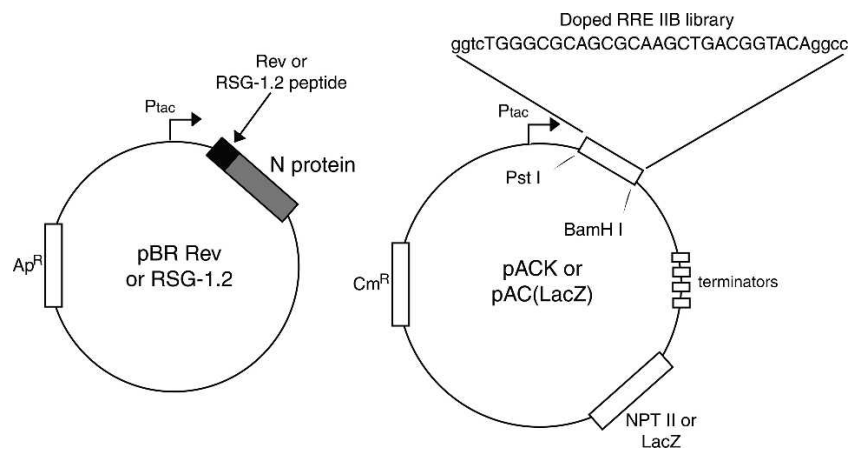

FIGURE 2. The two-plasmid system for detecting peptide-binding RRE variants. The pBR plasmid expresses Rev- or RSG-1.2 peptide fused to the $\mathrm{N}$ protein under the control of the tac promoter. In the pAC plasmid, the reporter transcript is driven from the tac promoter and contains the doped RRE library, followed by transcription terminators, and the reporter gene, NPT II or Lac Z, so that binding of either the Rev or RSG-1.2 peptide to the RRE variant results in transcription anti-termination and reporter gene expression. 
was doped at a frequency of $24 \%$ per residue, was prepared. This level of doping was expected to result in an average of five to six substitutions per sequence, and a representation of most of the RRE variants with two substitutions, including the $\mathrm{G}-\mathrm{G}$ to $\mathrm{A}-\mathrm{A}$ substitution observed in previous in vitro selections (Bartel et al. 1991; Giver et al. 1993), when $10^{5}$ sequences were screened (Harada and Frankel 1998).

In the primary selection, this doped library DNA was introduced into the pACK plasmid carrying the NPT II reporter and transformed into RSG-1.2 expressor cells (N567/pBR RSG-1.2) (Fig. 2; Peled-Zehavi et al. 2003). Of the estimated $2.3 \times 10^{6}$ transformants, $2.2 \%$ survival was observed on tryptone plates containing kanamycin $(5 \mu \mathrm{g} /$ $\mathrm{mL}$ ). In a secondary screen to eliminate reporter plasmidrelated false positives, the PCR-amplified library insert was reintroduced into the $\mathrm{pAC}$ plasmid carrying the $\mathrm{LacZ}$ reporter and retransformed into N567/pBR RSG-1.2 cells, and 9\% of the colonies exhibited blue color on plates containing X-gal. Reporter gene expression was scored by intensity of colony color (Harada et al. 1996; Harada and Frankel 1998), and those colonies with blue color similar to that of the wild-type RRE (scored as 4+) or darker were picked. Twenty-five individual clones were sequenced, and none showed anti-termination activity in BIV Tat expressor cells, indicating that false positives had been eliminated (Harada and Frankel 1998).

The selected clones could be divided into two classes depending on their specificity toward RSG-1.2 and Rev in the $\beta$-galactosidase colony color assay. Class I sequences possessed specificities similar to the wild-type sequence, namely, a colony color of $4+$ or more against RSG-1.2 and $3+$ or more against Rev, while the class II sequences showed low anti-termination activity against Rev (2+ or less) and were specific for RSG-1.2 (Table 1). In the class I clones, substitutions of nucleotides known to be important for Rev binding were not observed, with the exception of clone G1, with a G-G to A-A mismatch base pair in the internal loop (Bartel et al. 1991; Giver et al. 1993), and clone G17, with a substitution in the upper loop that was predicted to preserve the important bases for Rev binding, and most substitutions were in the terminal loop of the RRE. In the majority of the RSG-1.2-specific class II clones, substitutions in the upper stem at C51, U66, and G67 that are known to be important for Rev binding (clones G2, G5, G6, G9, G15, G18, G26, G30, G32, and G43) were observed. A number of clones that did not contain mutations in these three nucleotides, but contained substitutions that were expected to disrupt the secondary structure in this region, were also found (clones G3, G10, and G27). The secondary structures of representative class I and class II clones are shown in Figure 3.

\section{In vivo selection of Rev-specific RRE variants}

In an attempt to identify RRE variants specific for the Rev peptide using the same degenerate RRE library, selections/ screens to enrich Rev-binding clones were combined with a negative screen to eliminate RSG-1.2 binders. In the primary selection, the pACK plasmid carrying the doped RRE library was transformed into Rev expressor cells. Of the $1.5 \times 10^{6}$ transformants, $8 \%$ of the colonies survived on tryptone plates containing kanamycin $(5 \mu \mathrm{g} / \mathrm{mL})$. In a secondary screen to eliminate reporter plasmid-related false positives, the PCR-amplified library insert was reintroduced into the pAC plasmid carrying the LacZ reporter and retransformed into N567/pBR Rev cells. Ten percent of colonies exhibited blue color on plates containing X-gal. A total of 960 of those colonies with blue color similar to or darker than the wild-type RRE were pooled, and pAC plasmid was isolated. In a ternary screen to eliminate RSG-1.2-positive clones, the pool of selected pAC plasmids was transformed into RSG-1.2-expressing cells, and 288 white colonies were picked. In a quaternary screen to confirm activity against Rev, pAC plasmids were once again introduced into Rev expressor cells, and the sequences of 15 clones with varying degrees of intensity were determined, resulting in 13 unique sequences. The anti-termination activities of the selected RRE variants were tested against Rev and RSG-1.2 and were found to be specific for Rev as expected (Table 1). These clones were designated as class III sequences.

Nearly half of the clones (R3, R12, R26, R27, R28, R33, $\mathrm{R} 36$, and $\mathrm{R} 42$ ) were predicted to form a purine-rich internal loop in the upper stem as a result of a substitution at G50, C69, or A68, resembling Rev-binding RNAs selected in a previous study (Giver et al. 1993). A C69U substitution resulting in a G50-U69 wobble base pair was also observed in five clones (R5, R25, R29, R41, and R48). In addition, the $\mathrm{G}-\mathrm{G}$ to $\mathrm{A}-\mathrm{A}$ mismatch base pair in the internal loop was also observed in two clones (R12 and R34) (Bartel et al. 1991; Giver et al. 1993). The secondary structures of representative class III clones are shown in Figure 3. Taken together, these results confirmed the importance of the nucleotides important for Rev binding determined from previous studies (Fig. 1A; Bartel et al. 1991; Kjems et al. 1991, 1992; Tiley et al. 1992; Giver et al. 1993).

\section{In vitro peptide binding of the selected RRE variants}

To confirm that the anti-termination activities observed using the in vivo assay reflected the binding affinity of the RRE variants toward Rev and RSG-1.2, dissociation constants of representative RRE variants from each of the three classes shown in Figure 3 were determined by gel shift experiments (Fig. 4).

Two class I RRE variants, $\mathrm{G}^{\star}$ and G37, which showed slightly altered specificity toward Rev and RSG-1.2, were prepared. $\mathrm{G} 1^{\star}$ possesses the G48G71U72 to A48A71C72 mutation, but not the loop mutation of G1, which reported an increase in binding toward both Rev and RSG-1.2, while 
TABLE 1. Selected RSG-1.2- and Rev-binding RRE variants

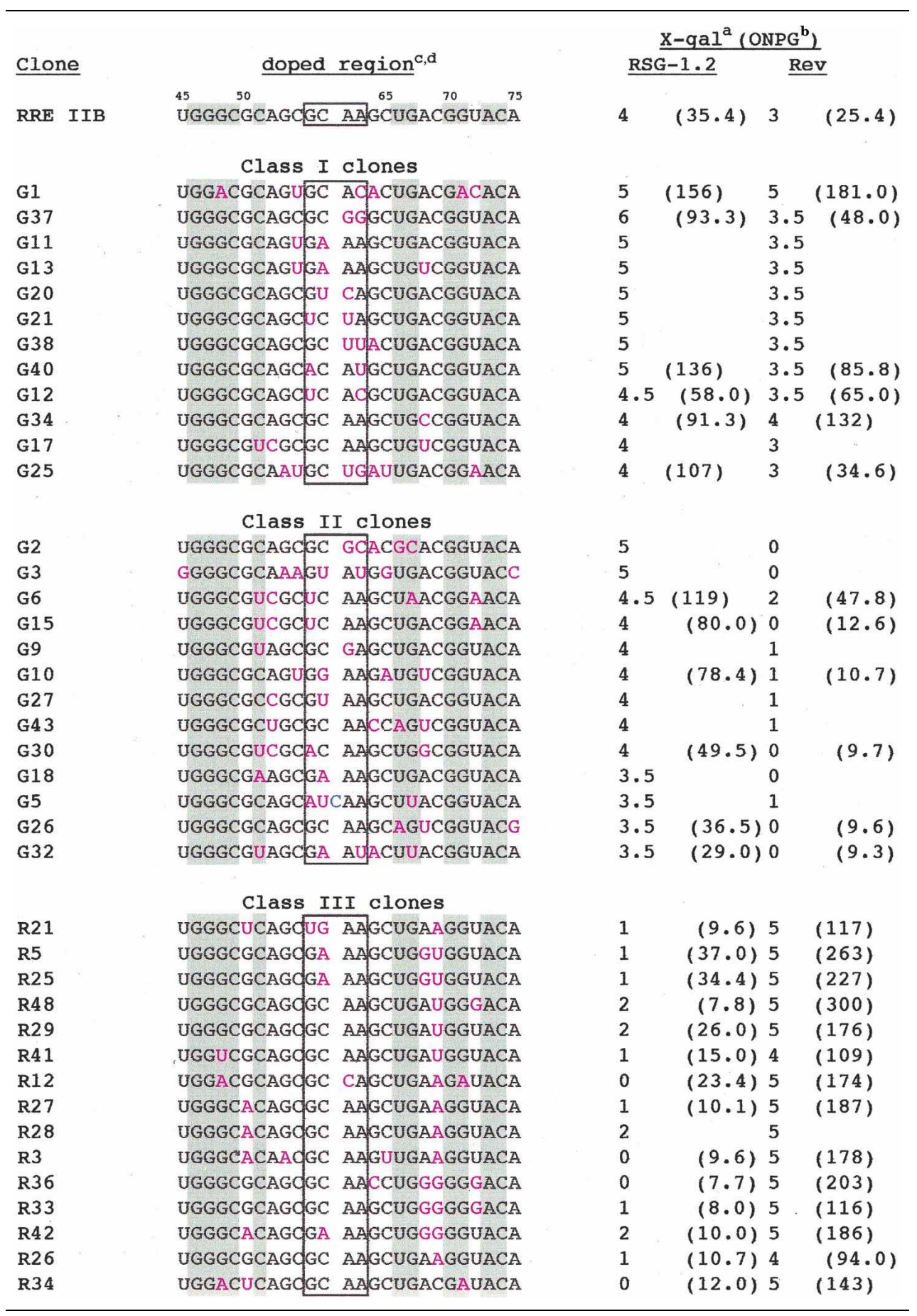

${ }^{a}$ Xgal colony color assays were performed as described (Peled-Zehavi et al. 2003). Numbers represent +'s used to score blue color intensity.

${ }^{\mathrm{b}} \beta$-galactosidase solution assays were performed as described (Peled-Zehavi et al. 2003).

${ }^{\mathrm{C}}$ The nucleotide sequence of the RRE used as a starting point for doped library design with the nucleotides known to be important for Rev-binding is shadowed (Bartel et al. 1991; Kjems et al. 1991, 1992; Tiley et al. 1992), and the tetraloop cap sequence is boxed.

${ }^{\mathrm{d}}$ The nucleotide sequences of the selected RRE variants with the nucleotides differing from the wild-type RRE are shown in pink, and the nucleotide insertions are shown in blue. 


\begin{tabular}{|c|c|c|c|c|c|c|}
\hline Class I & 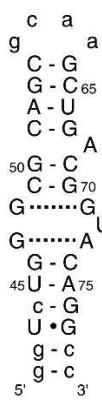 & $\begin{array}{l}\text { WT RRE } \\
\text { Rev 3+ } \\
\text { RSG } 4+\end{array}$ & 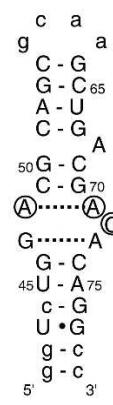 & $\begin{array}{l}\text { clone G1* } \\
\text { Rev 5+ } \\
\text { RSG 5+ }\end{array}$ & 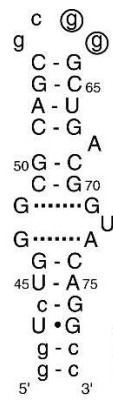 & $\begin{array}{l}\text { clone G37 } \\
\text { Rev } 3.5+ \\
\text { RSG } 6+\end{array}$ \\
\hline Class II & 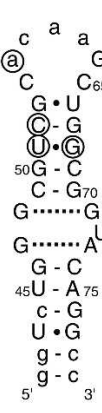 & $\begin{array}{l}\text { clone G30 } \\
\text { Rev - } \\
\text { RSG 4+ }\end{array}$ & 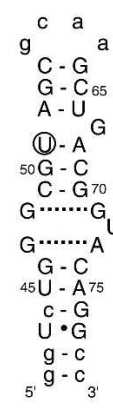 & $\begin{array}{l} \\
J \\
\text { clone G9 } \\
\text { (C51U) } \\
\text { Rev 1+ } \\
\text { RSG 4+ }\end{array}$ & 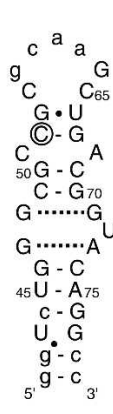 & $\begin{array}{l}\text { clone G27* } \\
\text { (A52C) } \\
\text { Rev 1+ } \\
\text { RSG 4+ }\end{array}$ \\
\hline Class III & 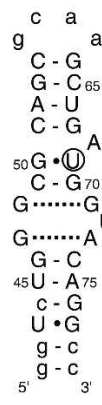 & $\begin{array}{l} \\
\text { clone R29 } \\
\text { (C69U) } \\
\text { Rev 5+ } \\
\text { RSG 2.5+ }\end{array}$ & 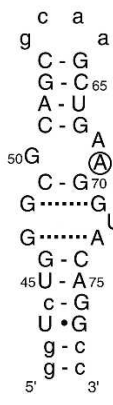 & $\begin{array}{l}\text { J } \\
\text { clone R26 } \\
\text { (C69A) } \\
\text { Rev } 4.5+ \\
\text { RSG 2+ }\end{array}$ & 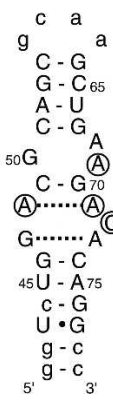 & $\begin{array}{l}\text { R26/G1* } \\
\text { hybrid }\end{array}$ \\
\hline
\end{tabular}

FIGURE 3. The predicted secondary structures of representative class I, class II, class III, and hybrid RRE variants, with nucleotide substitutions circled, and their anti-termination activities toward the Rev and RSG peptides indicated.

G37, which possesses an AA to GG mutation in the tetraloop, reported an increase in binding toward RSG-1.2, but not Rev, in vivo. Gel shift analysis of the peptide binding of $\mathrm{Gl}^{*}$ showed a twofold increase in the affinity toward Rev but not RSG-1.2, while that of G37 showed an approximately threefold increase in the affinity toward RSG-1.2, but only a small increase toward Rev, agreeing well with the in vivo activities observed.

Three class II RRE variants, G30, G9, and G27*, were tested for binding activity. G30, which contained four substitutions in the upper stem region that resulted in the formation of a five-base-pair stem capped by a seven-nucleotide loop bound 10-fold less strongly to Rev compared with WT RRE, while binding to RSG-1.2 remained almost the same. G9, which contained a C51U substitution, and G27*, which contained the A52C substitution observed in G27, were also tested for binding. In this case, while a decrease in binding toward Rev that was comparable to G30 was observed, the binding toward Rev also decreased somewhat. Nevertheless, a con- siderable shift in peptide-binding specificity toward RSG-1.2 was observed.

Two class III RRE variants, R26 and R29, with single nucleotide substitutions at position 69, were tested for peptide binding. R26 contained a C69A substitution that was predicted to form a three-nucleotide internal loop, while R29 contained a C69U substitution that presumably forms a wobble base pair with G50. The C69A and C69U substitutions showed a 100 -fold and 20 -fold decrease in the binding affinity toward RSG-1.2, respectively, while binding toward Rev remained the same, resulting in a large shift in peptide specificity toward Rev.

Next, in order to test whether the effect of the individual substitutions observed above on the peptide-binding specificity were additive, the A48A71C72 substitution of clone G1, which was found to increase the affinity of binding toward Rev, was combined with the C69A substitution of clone R26, which reduces binding toward RSG-1.2, to yield a G1*/R26 hybrid RNA. Unexpectedly, a reduction in the affinity toward the Rev peptide and a further reduction in
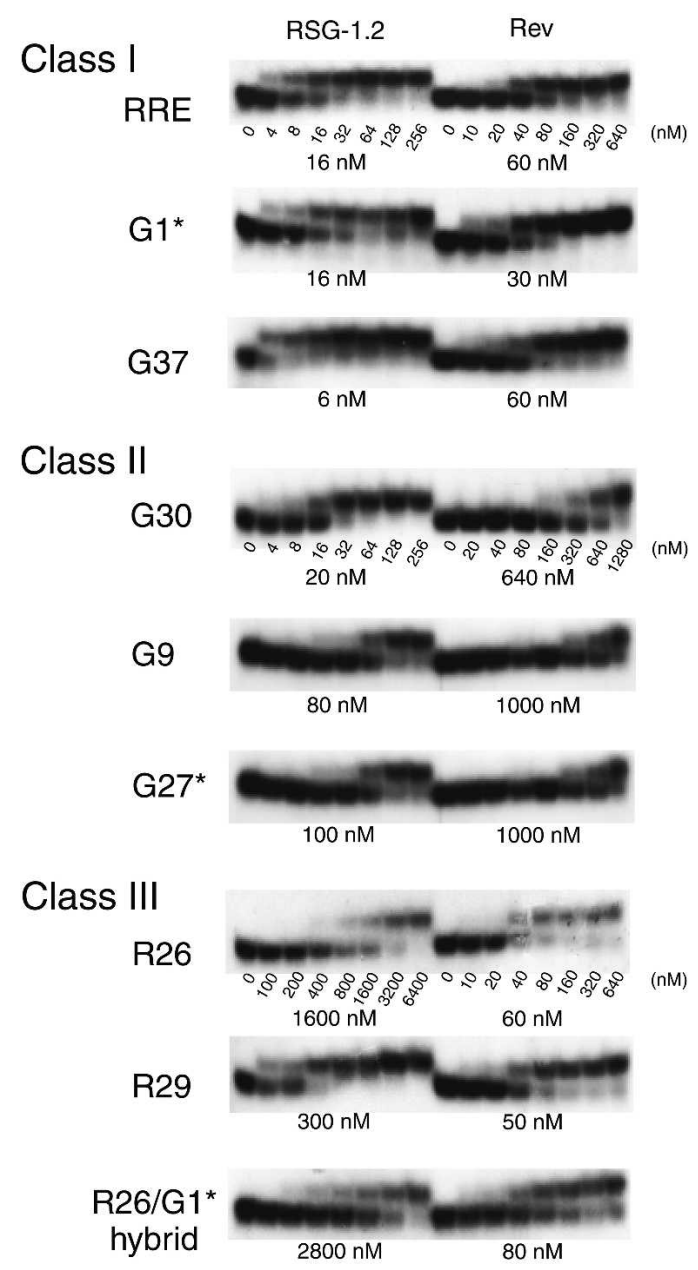

FIGURE 4. Gel shift analysis of the Rev- and RSG-1.2-binding activities of representative class I, class II, class III, and hybrid RRE variants. 
the affinity toward RSG-1.2 was observed, illustrating the difficulty in predicting the behavior of hybrid constructs.

\section{DISCUSSION}

\section{The difference in the nucleotide requirements for Rev and RSG-1.2 binding by the RRE}

Although the NMR structures of both the Rev-RRE and RSG-1.2-RRE complex have been determined (Battiste et al. 1996; Gosser et al. 2001; Zhang et al. 2001), biochemical studies are extremely informative in that they provide detailed information on the role and the importance of the individual molecular contacts observed. The nucleotide requirement for Rev binding determined from class I and class III sequences was in good agreement with previous biochemical studies and showed that three Watson-Crick base pairs, G46-C74, C49-G70, and C51-G67, and U66, as well as two non-Watson-Crick base pairs, G47-A73 and G48-G71, are important for binding (Figs. 1A, 5A). In addition, as in previously reported in vitro selection experiments (Bartel et al. 1991; Giver et al. 1993), a number of the selected RRE variants contained a substitution of the G48G71 base pair to the isostructural A48-A71 base pair.

In contrast, the nucleotides important for RSG-1.2 binding elucidated from class II sequences were localized in the vicinity of the internal loop, and nucleotides in the upper stem required for Rev binding (C51, U66, and G67) were not conserved (Fig. 5B). This agrees well with the fact that in the NMR structure of the Rev-RRE complex, the guanidinium group of R42 makes base-specific contacts with the C51-G67 base pair and U66, while in the RSG-1.2-RRE complex, only hydrophobic and electrostatic argininephosphate contacts are observed in this region (Gosser
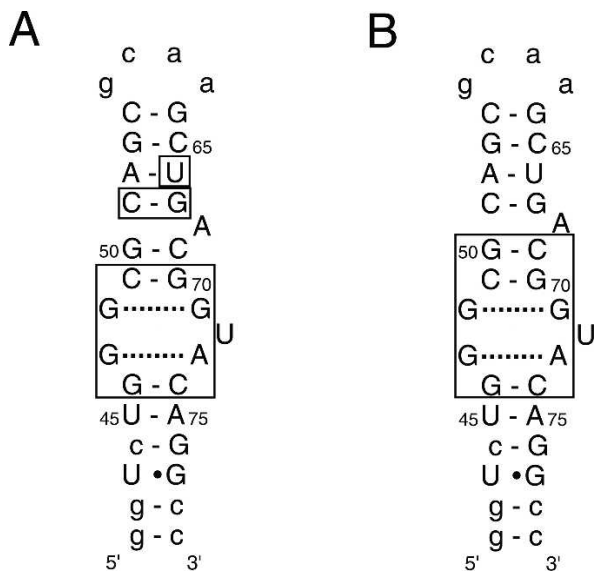

FIGURE 5. Primary and secondary structural elements important for Rev and RSG-1.2 binding. (A) Boxed nucleotides indicate those that were conserved in class I and class III clones and were predicted to be important for Rev binding. (B) Boxed nucleotides indicate nucleotides that were conserved in class I and class II sequences and were predicted to be important for RSG-1.2 binding. et al. 2001; Zhang et al. 2001). Another significant difference was the conservation of the G50-C69 base pair for RSG-1.2-binding class I and II clones, which has previously been shown not to be important for Rev binding (Bartel et al. 1991; Kjems et al. 1991, 1992; Tiley et al. 1992; Giver et al. 1993). The importance of this base pair for RSG-1.2 binding is further illustrated by the observation of substitutions to one or both of these bases in all of the Rev-specific class III sequences (Table 1). In the NMR structure of the RSG-1.2-RRE complex, the G50-C69 base pair appears to aid G70 in the recognition of the R14 side chain in a familiar simultaneous hydrogen bonding and stacking arrangement (Puglisi et al. 1992; Patel 1999), whereas the R14 guanidinium group, which is presumably hydrogen bonded to the Hoogsteen site of G70, is stacked with G50, and in turn results in the stacking of C69 with the R14 guanidinium group (Gosser et al. 2001; Zhang et al. 2001).

These results also served to confirm the utility of the bacterial in vivo assay in combinatorial analysis. While the number of sequences that can be tested in vivo is dependent on the number of transformants that can be obtained, and is limited to $\sim 10^{8}-10^{9}$ sequences, the above system is advantageous in that one can readily select for the affinity of choice, since anti-termination activity (kanamycin resistance or $\beta$-galactosidase activity) correlates with binding affinity.

\section{The molecular basis for the change in the peptide-binding specificity of RRE variants}

The peptide-binding specificity of the RRE variants identified in this study can be explained in terms of changes to the nucleotides shown to be important for Rev and RSG-1.2 binding shown in Figure 5. The bifunctional class I sequences did not contain substitution of the bases shown to be important for binding to both Rev and RSG-1.2, with the exception of the G48G71 to A48A71 substitution observed for clone G1, which has been shown to increase binding toward the Rev peptide (Bartel et al. 1991; Giver et al. 1993). Interestingly, gel shift analysis showed that clone G1 $\left(G 1^{\star}\right)$ bound twice as strongly to Rev, confirming previous results, while RSG-1.2 binding was the same as that of the WT RRE, resulting in a slight specificity shift toward Rev. Another class I clone, G37, which contained a mutation in the tetraloop capping the upper stem, showed a nearly threefold increase in binding for RSG-1.2, but only a slight increase for Rev binding, therefore leading to a moderate shift in specificity, in this case, toward RSG-1.2. This may be due to a long-range perturbation or tightening of the structure of the peptide binding site by the tetraloop structure that is relaxed by a mutation to a loop with normal stability. In the case of class II sequences, which are specific for RSG-1.2, substitutions to one or more of the bases in the upper stem that are important for Rev binding, C51, U66, and G67, were observed in 10 of the 13 clones, 
while the remaining three clones contained substitutions that were expected to disrupt the secondary structure of the upper stem. However, the decrease in the affinity for the Rev peptide was accompanied by a significant decrease in RSG-1.2 binding, illustrating how readily loss of function is likely to occur upon RNA evolution. Finally, in the case of the class III clones, substitutions at the G50-C69 base pair, which was found to be important for RSG-1.2 binding but not for Rev binding, were observed in every case, and resulted in the formation of a three-nucleotide bulge in seven of 13 sequences, and a G50-U69 wobble pair in four of 13 sequences.

The ability to tailor the specificity of the RRE toward the two peptides by combining the substitutions observed in this study would be an attractive approach to rational design. However, the results of the peptide binding of the $\mathrm{R} 26 / \mathrm{G1}^{*}$ hybrid show that, at least in this case, binding affinity is not additive. Presumably, the individual mutations cause slight perturbations of the structure of the RNA, which affect each other in various ways. A further understanding of the structure-function relationship of the individual mutations may be necessary to predict the peptidebinding specificities of such hybrid molecules.

\section{Evolvability of the mode of peptide binding by the RRE}

The potential to evolve the specificity or the mode of RNApeptide interactions in a stepwise manner without generating loss-of-function intermediates, as was the case in one study using the Rev-RRE system (Jain and Belasco 1996), has been suggested through studies on the Tat peptide-TAR RNA system (Smith et al. 1998, 2000). HIV and BIV Tat peptides bind specifically to what appear to be nearly identical cognate RNA sites, as observed in the NMR structures of the two Tat-TAR complexes; however, in an extended conformation and a $\beta$-turn conformation, respectively (Aboul-ela et al. 1995; Puglisi et al. 1995). A hybrid HIV/ BIV TAR that appeared to bind both BIV and HIV Tat peptides in the respective binding modes, and which may be regarded as a functional intermediate in the evolution of the two TARs, was identified, although it should be noted that high-affinity binding by the HIV Tat peptide required the presence of cyclin T1 (Smith et al. 1998). On the other hand, the JDV Tat peptide was found to bind to both the HIV, again only in the presence of cyclin T1, and BIV TARs in what appeared to be an extended and $\beta$-turn conformation, respectively (Smith et al. 2000). Therefore, the JDV Tat peptide may be regarded as a bifunctional intermediate in the switch of peptide-binding specificity. This suggested that stepwise mutation of an RNA via bifunctional intermediates may lead to the evolution of different binding modes.

In the present study, we have shown that single nucleotide changes in a bifunctional RNA, the RRE, are sufficient to switch not only the specificity, but also the mode of peptide binding, clearly demonstrating that it is indeed possible to evolve the peptide-binding strategy of an RNA in two discrete steps without intermediate loss of function. For example, the Rev-specific clone R26 can be evolved by a single nucleotide change (A69C) to the bifunctional RRE, which in turn can be evolved by a C51U mutation to the RSG-1.2-specific clone, G9, resulting in a total 330-fold change in specificity and a switch to a different mode of binding (Fig. 6).

Although the above results demonstrate the ease with which RNA is able to accommodate changes in binding mode, converse studies on the evolution the RNA-binding specificity of a peptide using the N-boxB system seem to indicate a somewhat different situation. First, it was shown by combinatorial analysis that single nucleotide substitutions in the $\lambda$ boxB loop could not be compensated by single amino acid changes in the $\lambda \mathrm{N}$ peptide (Franklin 1993). Furthermore, compensation for a single nucleotide change from the $\lambda$ boxB to P22 boxB was found to require five amino acid substitutions in the $\lambda \mathrm{N}$ peptide, with intermediate sequences showing weak function with both $\lambda$ and P22 boxB (Franklin 2004). This may be because the change in RNA-binding specificity is accompanied by a switch in the peptide-bound conformation of the RNA (Cai et al. 1998; Legault et al. 1998; Austin et al. 2003), which requires the concerted effect of several amino acid changes to support. It should, however, be noted that these studies were conducted using an in vivo functional assay that involves the formation of a multicomponent RNAprotein complex and may not reflect the peptide-box $\mathrm{B}$ interaction alone.

That the switch in the mode of peptide binding was observed in the RRE context by the RNA alone, and only

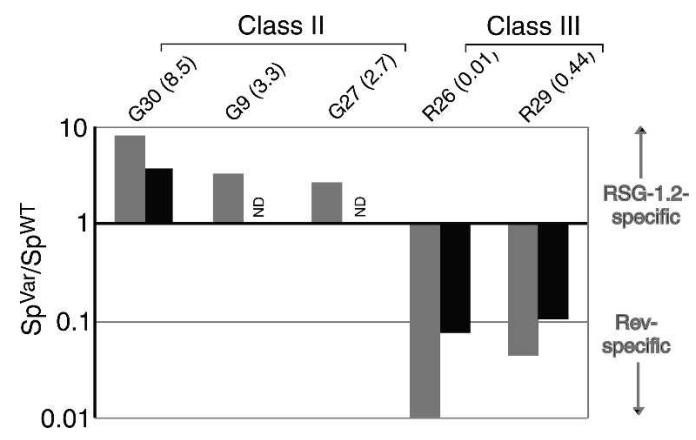

FIGURE 6. The change in peptide-binding specificity, $\mathrm{Sp}^{\mathrm{Var}} / \mathrm{Sp}^{\mathrm{WT}}$, of the selected RNA variants $\left(S p^{\text {Var }}\right)$ relative to that of WT RRE $\left(S p^{\text {WT }}\right)$. The change in specificity was determined using the following equation for the gel shift assay: $\left[\mathrm{Kd}_{\mathrm{var}}(\mathrm{Rev}) / \mathrm{Kd}_{\mathrm{var}}(\mathrm{RSG}-1.2)\right] /\left[\mathrm{Kd}_{\mathrm{RRE}}(\mathrm{Rev}) /\right.$ $\left.\mathrm{Kd}_{\text {RRE }}(\mathrm{RSG}-1.2)\right]$, where, for example, $\mathrm{Kd}_{\text {var }}(\mathrm{Rev})$ represents the $\mathrm{Kd}$ value of the RRE variant toward the Rev peptide and is shown as grey bars and values are shown in parentheses. The change in specificity was determined using the following equation for the $\beta$-gal (ONPG) assay: $\left[\mathrm{ONPG}_{\mathrm{var}}(\mathrm{RSG}-1.2) / \mathrm{ONPG}_{\mathrm{var}}(\mathrm{Rev})\right] /\left[\mathrm{ONPG} \mathrm{RRE}_{\mathrm{RSG}}(\mathrm{RS}-1.2) / \mathrm{ONPG}_{\mathrm{RRE}}\right.$ (Rev)], where, for example, ONPG var (RSG-1.2) represents the ONPG value of the RRE variant toward the RSG-1.2 peptide and is shown as black bars. 
in the presence of cyclin T1 in the TAR context, may reflect a difference in the structural flexibility of the two RNAs. RRE may more easily adapt its conformation to accommodate different peptide conformations due to its inherent flexibility, while the TAR may be unable to do so on its own and requires the assistance of cyclin T1. Indeed, NMR studies have shown that BIV TAR RNA alone forms a fairly rigid structure, with the Tat peptide adapting to the RNA structure (Puglisi et al. 1995). On the other hand, in the Rev-RRE system, a larger structural change in both the RNA and the peptide has been observed (Battiste et al. 1994; Tan and Frankel 1994; Peterson and Feigon 1996).

The adaptability of RNA to different modes of peptide binding presumably reflects the multidimensionality of conformational space that a given RNA has available for peptide recognition (Leulliot and Varani 2001). The evolvability of RNA-peptide complexes described here was likely to have been utilized throughout the evolution of RNA-protein complexes, starting from the transition from an RNA world to an RNA-protein world, and leading to the diverse structures and biological functions observed in contemporary organisms and viruses. An understanding of the mechanism underlying this process may also provide insights on the evolution of viral RNA-protein interactions and their role in the emergence of novel RNA viruses and drug resistance.

\section{MATERIALS AND METHODS}

\section{Construction of the doped RRE library}

The following oligonucleotide encoding the $\lambda$ boxA element followed by a degenerate RRE region and flanked at the $5^{\prime}$ - and $3^{\prime}$-side by Pst I and BamH I sites, respectively, was synthesized on a Cyclone Plus DNA Synthesizer (MilliGen/Bioresearch): 5' gggCTGCAGGTCGACGCTCTTAAAAATTAAGGTCTGGGCGCA GCGCAAGCTGACGGTACAGGCCAGCATTCAAAGCAGGGATC Cccg- $3^{\prime}$, where the degenerate region (underlined) contained the base indicated at $76 \%$ frequency and each of the remaining three bases at a frequency of $8 \%$ each. A primer (5'-CGGGGATCCC TGCTTTGAATGC- $3^{\prime}$ ) was annealed to the $3^{\prime}$-end of the library oligonucleotide, second-strand synthesis was carried out using Sequenase 2.0 polymerase (USB), and the resulting double-stranded DNA was digested using Pst I and BamH I.

\section{Selection of RRE variants with RSG-1.2-binding activity}

The procedures for the in vivo selection were based on the previously described methods for peptide selection (Peled-Zehavi et al. 2003). In the primary selection, the doped RRE library plasmid was prepared by ligation of $0.15 \mu \mathrm{g}$ of library insert into $1.5 \mu \mathrm{g}$ of pACK-nut ${ }^{-}$reporter plasmid, followed by phenol extraction and ethanol precipitation. This DNA was used to transform N567 cells containing pBR RSG-1.2 (N567/ pBR RSG-1.2) by electroporation and spread onto tryptone plates containing $5 \mu \mathrm{g} /$ $\mathrm{mL}$ kanamycin, and incubated for $68 \mathrm{~h}$ at $21^{\circ} \mathrm{C}$. Of the estimated total of $2.3 \times 10^{6}$ transformants obtained, $5.5 \times 10^{4}$ colonies
(2.2\% survival) were observed on plates. Colonies were scraped off plates using tryptone broth containing antibiotics, and plasmid DNA was isolated from the collected cells. The library region of the selected pAC plasmid was amplified by PCR using a pAC forward primer ( $5^{\prime}$-GGCTTATCGAAATTAATAC- $\left.3^{\prime}\right)$ and reverse primer $\left(5^{\prime}\right.$-ACGG TAAGAGTGCCAGTG- $\left.3^{\prime}\right)$. The amplified library fragment was then digested using Pst I and BamH I, phenol extracted, and purified on an $8 \%$ polyacrylamide gel. In the secondary screen, this insert was ligated into the Pst I and BamH I site of the $\mathrm{pAC}(\mathrm{LacZ})$ nut $^{-}$reporter plasmid and used to transform N567/ pBR RSG-1.2 cells by heat shock. The cells were spread onto plates containing $80 \mu \mathrm{g} / \mathrm{mL} \mathrm{X-gal} \mathrm{and} \mathrm{incubated} \mathrm{for} 20 \mathrm{~h}$ at $37^{\circ} \mathrm{C}$, and $\sim 9 \%$ of the colonies turned blue. Reporter gene expression was scored by intensity of colony color and by ONPG assays as described previously (Harada et al. 1996, 1997). Plasmid DNA from 48 individual colonies with blue color comparable or darker than the WT RRE (scored as 4+) were isolated and the library region of the pAC plasmids were individually PCR amplified and reintroduced into the $\mathrm{pAC}$ nut $^{-}$reporter plasmid as described above and subjected to a tertiary screen to confirm activity in N567/ pBR RSG-1.2 cells, of which the sequence of 25 clones with colony color of $4+$ or higher was determined.

\section{Selection of RRE variants specific for Rev peptide}

In the primary selection, the same doped RRE library plasmid was used to transform N567/ pBR Rev cells by electroporation and spread onto tryptone plates containing $5 \mu \mathrm{g} / \mathrm{mL}$ of kanamycin. Plates were incubated for $68 \mathrm{~h}$ at $23^{\circ} \mathrm{C}$, and of the total of $1.5 \times 10^{6}$ transformants, 1.2 times $10^{5}$ colonies were observed on plates. Plasmid DNA from the pooled cells were PCR amplified, digested with Pst I and BamH I, and gel purified as described above. In the secondary screen, the library insert was ligated into the Pst I and $\mathrm{BamH}$ I site of the $\mathrm{pAC}(\mathrm{LacZ})$ nut $^{-}$reporter plasmid and used to transform N567/ pBR Rev cells by heat shock. The cells were spread onto plates containing $80 \mu \mathrm{g} / \mathrm{mL}$ X-gal and incubated for 20 $\mathrm{h}$ at $37^{\circ} \mathrm{C}$ and $4.3 \times 10^{4}$ colonies were observed, of which $10 \%$ were blue. Nine hundred sixty blue colonies (divided into three classes according to colony color $5+, 4+$, and $3+$ ) were picked and individually incubated for $16 \mathrm{~h}$ at $37^{\circ} \mathrm{C}$ in tryptone containing ampicillin and chloramphenicol in 96 well plates. In the ternary screen, plasmid isolated from the three separate pooled colonies was used to transform N567/ pBR RSG-1.2 cells by heat shock, spread onto Xgal plates, and incubated for $20 \mathrm{~h}$ at $37^{\circ} \mathrm{C}$. Of the total $\sim 4000$ colonies in each case, approximately half were white. Ninety-six white colonies were picked from each group and plasmid DNA was isolated. In the quaternary screen, plasmid DNA from the three groups were transformed into N567/ pBR Rev cells by heat shock, and of the 10,000 colonies obtained, an average of $14 \%$ were blue. The sequence of 15 clones was determined.

\section{Peptide-binding assays}

Rev and RSG-1.2 peptides were prepared as previously described (Harada et al. 1997). Internally labeled RNAs were transcribed in vitro using T7 RNA polymerase and $\left[\alpha-{ }^{32} \mathrm{P}\right] \mathrm{CTP}$ (Perkin Elmer, $3000 \mathrm{Ci} / \mathrm{mmol}$ ) as described (Chen and Frankel 1994). Gel shift assays were carried out by incubating the internally labeled RNA (0.5-1 nM) with varying amounts of Rev or RSG-1.2 peptide at 
$4^{\circ} \mathrm{C}$ in $10-\mu \mathrm{L}$ binding mixtures containing $10 \mathrm{mM}$ HEPES-KOH

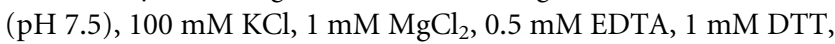
$50 \mu \mathrm{g} / \mathrm{mL}$ tRNA, and $10 \%$ glycerol, and resolving RNA and RNApeptide complexes on $10 \%$ polyacrylamide, $0.5 \times$ TBE $(45 \mathrm{mM}$ Tris/45 mM boric acid/1 mM EDTA, $\mathrm{pH} 8$ ) gels at $4^{\circ} \mathrm{C}$. Apparent $\mathrm{Kd}$ is defined as the concentration of peptide required to shift $50 \%$ of the free RNA into the complex.

\section{ACKNOWLEDGMENTS}

We thank Satoru Horiya, Colin Smith, and Alan Frankel for critical comments on the manuscript. This work was supported by a Grant-in-Aid for Scientific Research in Priority Areas from the Ministry of Education, Culture, Sports, Science and Technology (MEXT) of Japan.

Received March 10, 2005; accepted May 30, 2005.

\section{REFERENCES}

Aboul-ela, F., Karn, J., and Varani, G. 1995. The structure of the human immunodeficiency virus type-1 TAR RNA reveals principles of RNA recognition by Tat protein. J. Mol. Biol. 253: 313332.

Austin, R.J., Xia, T., Ren, J., Takahashi, T.T., and Roberts, R.W. 2003. Differential modes of recognition in $\mathrm{N}$ peptide-boxB complexes. Biochemistry 42: 14957-14967.

Bartel, D.P., Zapp, M.L., Green, M.R., and Szostak, J.W. 1991. HIV-1 Rev regulation involves recognition of non-Watson-Crick base pairs in viral RNA. Cell 67: 529-536.

Battiste, J.L., Tan, R., Frankel, A.D., and Williamson, J.R. 1994. Binding of an HIV Rev peptide to Rev responsive element RNA induces formation of purine-purine base pairs. Biochemistry 33: 2741-2747.

Battiste, J.L., Mao, H., Rao, N.S., Tan, R., Muhandiram, D.R., Kay, L.E., Frankel, A.D., and Williamson J.R. 1996. $\alpha$ Helix-RNA major groove recognition in an HIV-1 Rev peptide-RRE RNA complex. Science 273: 1547-1551.

Cai, Z., Gorin, A., Frederick, R., Ye, X., Hu, W., Majumdar, A., Kettani, A., and Patel, D.J. 1998. Solution structure of P22 transcriptional antitermination $\mathrm{N}$ peptide-boxB RNA complex. Nat. Struct. Biol. 5: 203-212.

Calnan, B.J., Biancalana, S., Hudson, D., and Frankel, A.D. 1991. Analysis of arginine-rich peptides from the HIV Tat protein reveals unusual features of RNA-protein recognition. Genes \& Dev. 5: 201-210.

Chen, L. and Frankel, A.D. 1994. An RNA-binding peptide from bovine immunodeficiency virus Tat protein recognizes an unusual RNA structure. Biochemistry 33: 2708-2715.

- 1995. A peptide interaction in the major groove of RNA resembles protein interactions in the minor groove of DNA. Proc. Natl. Acad. Sci. 92: 5077-5081.

Cheng, A.C., Calabro, V., and Frankel, A.D. 2001. Design of RNAbinding proteins and ligands. Curr. Opin. Struct. Biol. 11: 478-484.

Das, C. and Frankel, A.D. 2003. Sequence and structure space of RNAbinding peptides. Biopolymers 70: $80-85$.

Frankel, A.D. 2000. Fitting peptides into the RNA world. Curr. Opin. Struct. Biol. 10: 332-340.

Frankel, A.D. and Smith, C.A. 1998. Induced folding in RNA-protein recognition: More than a simple molecular handshake. Cell 92: 149-151.

Franklin, N.C. 1993. Clustered arginine residues of bacteriophage $\lambda \mathrm{N}$ protein are essential to antitermination of transcription, but their locale cannot compensate for boxB loop defects. J. Mol. Biol. 231: 343-360.
2004. Morphing molecular specificities between Arm-peptide and NUT-RNA in the antitermination complexes of bacteriophages $\lambda$ and P22. Mol. Microbiol. 52: $815-822$.

Friesen, W.J. and Darby, M.K. 1998. Specific RNA binding proteins constructed from zinc fingers. Nat. Struct. Biol. 5: 543546.

Giver, L., Bartel, D., Zapp, M., Pawul, A., Green, M., and Ellington, A.D. 1993. Selective optimization of the Rev-binding element of HIV-1. Nucleic Acids Res. 21: 5509-5516.

Gosser, Y., Hermann, T., Majumdar, A., Hu, W., Frederick, R., Jiang, F., Xu, W., and Patel, D.J. 2001. Peptide-triggered conformational switch in HIV-1 RRE RNA complexes. Nat. Struct. Biol. 8: 146150.

Harada, K. and Frankel, A.D. 1998. In vivo selection of specific RNAbinding polypeptides using a transcriptional antitermination reporter assay. In RNA-protein interactions: A practical approach (ed. C.W.J. Smith), pp. 217-236. Oxford University Press, Oxford, UK.

Harada, K., Martin, S.S., and Frankel, A.D. 1996. Selection of RNAbinding peptides in vivo. Nature 380: 175-179.

Harada, K., Martin, S.S., Tan, R., and Frankel, A.D. 1997. Molding a peptide into an RNA site by in vivo peptide evolution. Proc. Natl. Acad. Sci. 94: 11887-11892.

Heaphy, S., Finch, J.T., Gait, M.J., Karn, J., and Singh, M. 1991. Human immunodeficiency virus type 1 regulator of virion expression, rev, forms nucleoprotein filaments after binding to a purinerich "bubble" located within the rev-responsive region of viral mRNAs. Proc. Natl. Acad. Sci. 88: 7366-7370.

Jain, C. and Belasco, J.G. 1996. A structural model for the HIV-1 RevRRE complex deduced from altered-specificity Rev variants isolated by a rapid genetic strategy. Cell 87: 115-125.

Kjems, J., Brown, M., Chang, D.D., and Sharp, P.A. 1991. Structural analysis of the interaction between the human immunodeficiency virus Rev protein and the Rev response element. Proc. Natl. Acad. Sci. 88: 683-687.

Kjems, J., Calnan, B.J., Frankel, A.D., and Sharp, P.A. 1992. Specific binding of a basic peptide from HIV-1 Rev. EMBO J. 11: 11191129.

Legault, P., Li, J., Mogridge, J., Kay, L.E., and Greenblatt, J. 1998. NMR structure of the bacteriophage $\lambda \mathrm{N}$ peptide/boxB RNA complex: Recognition of a GNRA fold by an arginine-rich motif. Cell 93: 289-299.

Leulliot, N. and Varani, G. 2001. Current topics in RNA-protein recognition: Control of specificity and biological function through induced fit and conformational capture. Biochemistry 40: 79477956.

Patel, D.J. 1999. Adaptive recognition in RNA complexes with peptides and protein modules. Curr. Opin. Struct. Biol. 9: 7487.

Peled-Zehavi, H., Horiya, S., Das, C., Harada, K., and Frankel, A.D. 2003. Selection of RRE RNA binding peptides using a kanamycin antitermination assay. RNA 9: 252-261.

Peterson, R.D. and Feigon, J. 1996. Structural change in Rev responsive element RNA of HIV-1 on binding Rev peptide. J. Mol. Biol. 264: 863-877.

Puglisi, J.D., Tan, R., Calnan, B.J., Frankel, A.D., and Williamson, J.R. 1992. Conformation of the TAR RNA-arginine complex by NMR spectroscopy. Science 257: 76-80.

Puglisi, J.D., Chen, L., Blanchard, S., and Frankel, A.D. 1995. Solution structure of a bovine immunodeficiency virus Tat-TAR peptideRNA complex. Science 270: 1200-1203.

Smith, C.A., Crotty, S., Harada, Y., and Frankel, A.D. 1998. Altering the context of an RNA bulge switches the binding specificities of two viral Tat proteins. Biochemistry 37: 10808-10814.

Smith, C.A., Calabro, V., and Frankel, A.D. 2000. An RNA-binding chameleon. Mol. Cell 6: 1067-1076.

Tan, R. and Frankel, A.D. 1994. Costabilization of peptide and RNA structure in an HIV Rev peptide-RRE complex. Biochemistry 33: 14579-14585. 
1998. A novel glutamine-RNA interaction identified by screening libraries in mammalian cells. Proc. Natl. Acad. Sci. 95: $4247-4252$.

Tan, R., Chen, L. Buettner, J.A., Hudson, D., and Frankel, A.D. 1993. RNA recognition by an isolated $\alpha$-helix. Cell 73: 10311040.

Tiley, L.S., Malim, M.H., Tewary, H.K., Stockley, P.G., and Cullen, B.R. 1992. Identification of a high-affinity RNA-binding site for the human immunodeficiency virus type $1 \mathrm{Rev}$ protein. Proc. Natl. Acad. Sci. 89: 758-762.

Weeks, K.M. and Crothers, D.M. 1993. Major groove accessibility of RNA. Science 261: 1574-1577.

Zhang, Q., Harada, K., Cho, H.S., Frankel, A.D., and Wemmer, D.E. 2001. Structural characterization of the complex of the Rev response element RNA with a selected peptide. Chem. Biol. 8: 511-520. 

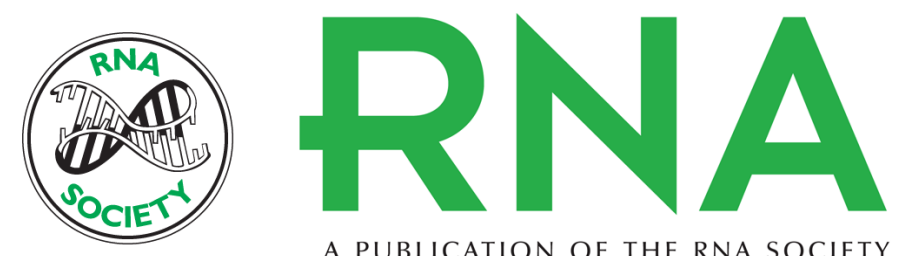

A PUBLICATION OF THE RNA SOCIETY

\section{Evolvability of the mode of peptide binding by an RNA}

\section{TETSUYA IWAZAKI, XIANGLAN LI and KAZUO HARADA}

RNA 2005 11: 1364-1373

References This article cites 39 articles, 12 of which can be accessed free at: http://rnajournal.cshlp.org/content/11/9/1364.full.html\#ref-list-1

License

Email Alerting Receive free email alerts when new articles cite this article - sign up in the box at the Service top right corner of the article or click here. 